\title{
Stomach Perforation Caused by Ingesting Liquid Nitrogen: A Case Report on the Effect of a Dangerous Snack
}

\author{
Dong-Wook Kim \\ Department of Surgery, Dankook University Hospital, Cheonan, Korea
}

We report our experience with a case of stomach perforation after accidental ingestion of liquid nitrogen. A 13-year-old boy ate a snack at an amusement park and began to complain of sudden onset of severe abdominal pain with shortness of breath. It was determined that the snack he had ingested had been cooled with liquid nitrogen. A computed tomography scan of the abdomen and a chest $\mathrm{X}$-ray showed a large volume of pneumoperitoneum. During surgery, a 4-cm perforation of the angularis incisura of the stomach was identified. Primary repair and omentopexy was performed. The patient was discharged without postoperative complications. Clin Endosc 2018;51:381-383

Key Words: Stomach perforation; Liquid nitrogen; Barotrauma

\section{INTRODUCTION}

Liquid nitrogen is used as an additive to food and beverages to cool them and create an aesthetic "smoking" effect. Ingestion of liquid nitrogen occurs infrequently, but it may create fatal damage in the gastrointestinal tract. The cause of injury can be explained by two mechanisms: cold/freezing injury upon contact with mucosal surfaces and rapid volume expansion as nitrogen gas is formed. We present the case of a 13-year-old boy who accidentally ingested liquid nitrogen and suffered serious gastrointestinal consequences.

\section{CASE REPORT}

A 13-year-old boy was transferred to the emergency room

Received: November 2, 2017 Revised: January 19, 2018

Accepted: January 23, 2018

Correspondence: Dong-Wook Kim

Department of Surgery, Dankook University Hospital, 201 Manghyang-ro, Dongnam-gu, Cheonan 31116, Korea

Tel: +82-41-550-7688, Fax: +82-41-550-4055, E-mail: twdw82@dkuh.co.kr ORCID: https://orcid.org/0000-0002-3552-5988

(c) This is an Open Access article distributed under the terms of the Creative Commons Attribution Non-Commercial License (http://creativecommons.org/ licenses/by-nc/3.0) which permits unrestricted non-commercial use, distribution, and reproduction in any medium, provided the original work is properly cited. shortly after falling down at an amusement park. He had eaten a snack to which liquid nitrogen had been added for rapid cooling, and his parents had witnessed the incident. The patient complained of sudden onset of severe abdominal pain and shortness of breath immediately after ingestion. There were no symptoms of dysphagia or sore throat. He had no remarkable medical or surgical history.

On examination, his blood pressure was $140 / 90 \mathrm{~mm} \mathrm{Hg}$, heart rate was 120 beats $/ \mathrm{min}$, body temperature was $37.2^{\circ} \mathrm{C}$, respiratory rate was 20 breaths/min, and oxygen saturation at room air was $98 \%$. He was not clinically intoxicated. There was no injury to the head, nose, or throat identified on examination, and the chest was clear to auscultation bilaterally. The abdomen was tense and severely distended with tenderness (Fig. 1). An erect chest X-ray (Fig. 2A) showed a large volume of pneumoperitoneum. A computed tomography scan of his abdomen confirmed the presence of a large volume of pneumoperitoneum, but the site of perforation could not be precisely located (Fig. 2B). His white blood cell count was $21,400 / \mu \mathrm{L}$, but the results for other blood count parameters were normal, as were the results for blood chemistry and electrolytes.

The patient was immediately taken to the operating room for an exploratory laparotomy, in which he was found to have a large amount of gas under tension. There were multiple erythemas along the lesser curvature of the stomach, but no sign 

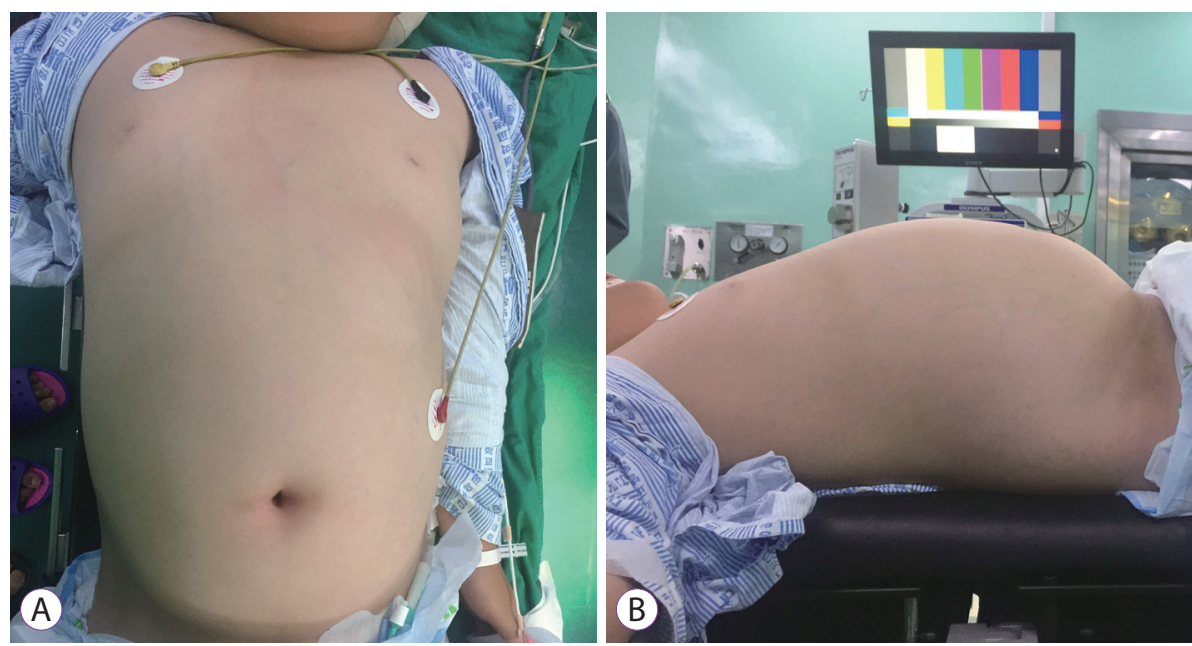

Fig. 1. The abdomen of the 13-year-old boy was severely distended. (A) Front view. (B) Side view.
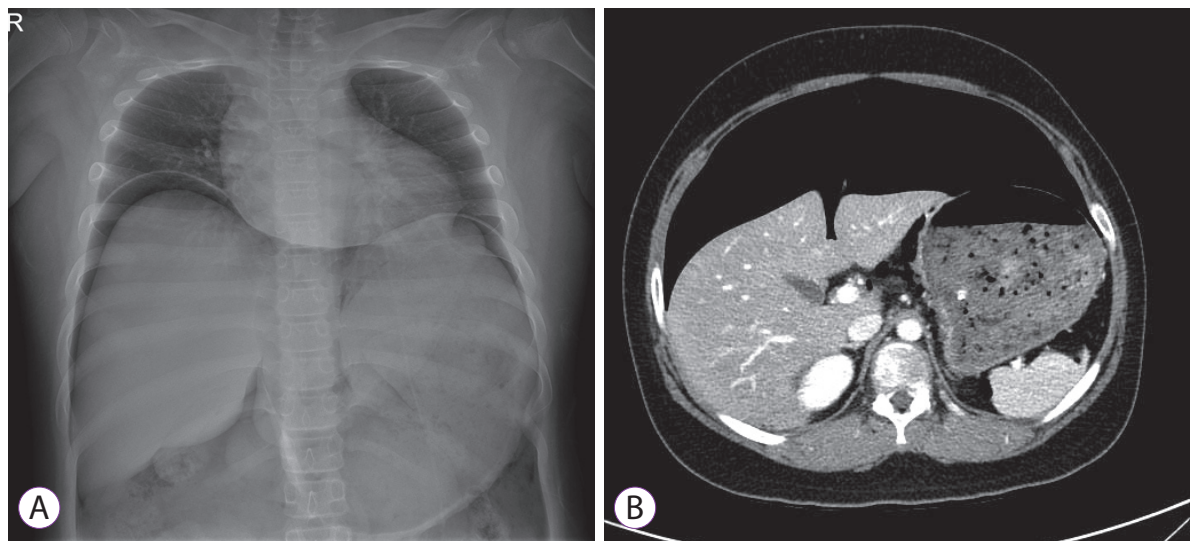

Fig. 2. Massive pneumoperitoneum of the abdomen on (A) chest X-ray and (B) computed axial tomography scan.

of prominent peritonitis. The rest of the abdominal contents were normal. Following precise, repeated observation, we located air bubbles around the gastrohepatic ligament. After dividing the gastrohepatic ligament of the stomach, a $4-\mathrm{cm}$ linear perforation was identified in the angularis incisura (Fig. 3). A large amount of food was also found. There was no evidence of tissue necrosis or hemorrhage in the other parts of the stomach. Primary repair was performed in layers with omentopexy.

The patient was extubated and transferred to the intensive care unit for close observation. He was transferred to the general ward on the second postoperative day. Oral intake was started on the fourth postoperative day, and there were no immediate complications. There were no abnormal findings, such as esophageal injury or achalasia (Fig. 4). The patient was discharged 8 days after the surgery. At a follow-up appointment after discharge, the patient was doing well without any symptoms.

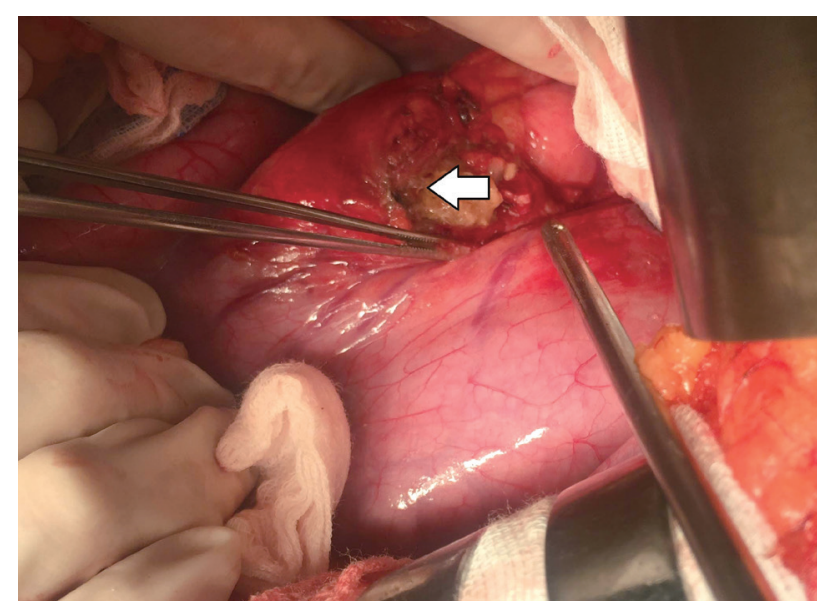

Fig. 3. A 4-cm perforation in the angularis incisura of the stomach was identified.

\section{DISCUSSION}

Nitrogen is an inert, odorless, and colorless gas in the air that does not react with other air molecules such as oxygen. It 


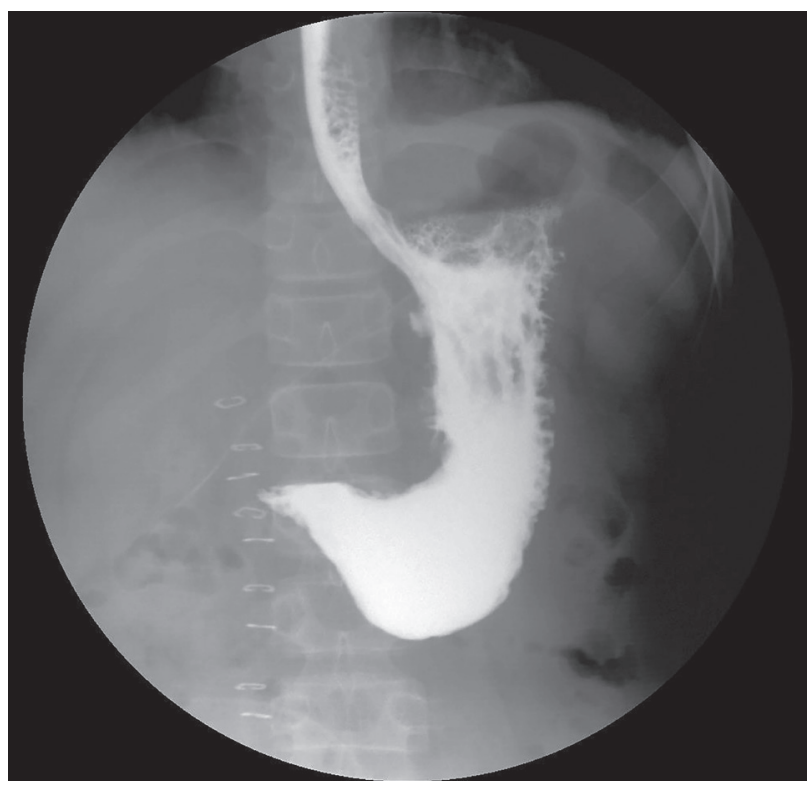

Fig. 4. Postoperative upper gastrointestinal series.

exists only as a gas in its natural state. Liquid nitrogen is a colorless liquid that can be obtained by compressing nitrogen gas to a certain pressure and then cooling it. It has many scientific, medical, and dietary uses as a coolant or freezing agent. Due to its extremely low boiling point $\left(-196^{\circ} \mathrm{C}\right)$, liquid nitrogen rapidly evaporates when exposed to room temperature, freezing anything it comes into contact with and generating a white smoke. ${ }^{1,2}$

Accidents caused by liquid nitrogen have often been reported. Most of them have been related to external exposure and subsequent cold injury occurring in a laboratory setting. ${ }^{3}$ There have been a few cases similar to the accident reported herein throughout the world. In all previous cases, the clinical presentation was almost the same as in our case: a rapid onset of abdominal pain accompanied by respiratory distress after ingestion. In addition, the site of perforation was confirmed to be over the lesser curvature of the stomach, as in our case. There was no damage to the gastrointestinal tract mucosa in most cases. ${ }^{1,2}$

This phenomenon can be explained by the mechanism of barotrauma. In other words, rapid expansion of the evaporating liquid nitrogen is the most significant cause of injury. Liquid nitrogen has a volume ratio of 1:694 (liquid:gas), which generates a large amount of gas even in small quantities. This volume expansion contributes to the rapid distension of any hollow viscus and ultimately causes perforation with a large volume of pneumoperitoneum. ${ }^{4,5}$
A pediatric emergency report documents why the perforation always occurs in the lesser curvature. Berrizbeitia et al. ${ }^{1}$ described this phenomenon using Laplace's law: the larger the sphere radius, the larger the wall tension required to withstand a given internal pressure. The body of the stomach can distend freely, as compared with the fundus or antrum; therefore, it is the weakest point of the wall under tension. Moreover, the rupture tends to occur along the lesser curvature where the stomach is relatively fixed by the celiac trunk, the left gastric artery, and the gastroesophageal junction. ${ }^{1}$

To the best of our knowledge, this is the first of such cases reported in Asia. In most other cases in different countries, a total gastrectomy or at least a feeding jejunostomy was performed. ${ }^{1,4}$ Although abdominal compartment syndrome was suspected and could not be approached using laparoscopy, this patient fortunately recovered without any problems after primary repair.

The snack that caused the incident was a cookie that emits "smoke" like a dragon. This interesting phenomenon is popular with young children, and they are able to buy the snack easily, without restriction. As seen in this case, it can be hazardous to accidentally ingest liquid nitrogen. Our 13-year-old patient was in critical condition and has been left with a $20-\mathrm{cm}$ scar on his abdomen. Currently, the Food and Drug Administration is reviewing the safety standards for using liquid nitrogen in food.

In conclusion, the ingestion of liquid nitrogen can be fatal, as it may cause gastrointestinal injury. The primary cause of perforation is barotrauma. Immediate surgical intervention is critical to treat the pneumoperitoneum and locate the perforation site.

\section{Conflicts of Interest}

The author has no financial conflicts of interest.

\section{REFERENCES}

1. Berrizbeitia LD, Calello DP, Dhir N, O’Reilly C, Marcus S. Liquid nitrogen ingestion followed by gastric perforation. Pediatr Emerg Care 2010;26:48-50.

2. Walsh MJ, Tharratt SR, Offerman SR. Liquid nitrogen ingestion leading to massive pneumoperitoneum without identifiable gastrointestinal perforation. J Emerg Med 2010;38:607-609.

3. Gill JR, Ely SF, Hua Z. Environmental gas displacement: three accidental deaths in the workplace. Am J Forensic Med Pathol 2002;23:26-30.

4. Pollard JS, Simpson JE, Bukhari MI. A lethal cocktail: gastric perforation following liquid nitrogen ingestion. BMJ Case Rep 2013;2013.

5. Koplewitz BZ, Daneman A, Fracr S, Ein SH, McGuigan MA, Mian M. Gastric perforation attributable to liquid nitrogen ingestion. Pediatrics 2000;105(1 Pt 1):121-123. 